\title{
How to Manage Your Inbox: Is a Once a Day Strategy Best?
}

\author{
Adam Bradley $^{1} \quad$ Duncan P. Brumby ${ }^{1 *}$ \\ ${ }^{1} \mathrm{UCL}$ Interaction Centre \\ University College London \\ London WC1E 6BT UK \\ *Brumby@cs.ucl.ac.uk \\ Anna L. Cox ${ }^{1} \quad$ Jon Bird ${ }^{2}$ \\ ${ }^{2}$ Department of Computing \\ City University London \\ London EC1V OHB UK
}

ch.

\begin{abstract}
Many people are overloaded by the amount of email they receive. Because of this, a considerable amount of time can be spent every day managing one's inbox. Previous work has shown that people adopt different strategies for managing their inbox. However, there has been little work examining how the choice of email management strategy impacts the total time that one gives to email activities each day. In the current study seven academics spent one week trying out different email management strategies: either a once-a-day strategy or a frequent strategy. Data on the amount of time spent managing email and subjective feelings towards each strategy were gathered. Results suggest that a once a day email management strategy may be effective in reducing the total time spent dealing with email.
\end{abstract}

Email overload, email strategies, personal informatics, work-life balance.

\section{INTRODUCTION}

Email is a ubiquitous form of communication. It is the second most widely used method of communication in business and academic institutions, after talking face to face (Crystal, 2001). There are many benefits to using email as a communication tool: it is fast, low cost, works across long distances, and is asynchronous. It can also support group messaging threads, mailing lists and task management for many users (Whittaker, Bellotti, \& Gwizdka, 2006).

There are drawbacks to email - many people receive more messages than they can easily manage. They find themselves in a situation where regardless of the amount of time they spend managing their inbox they are unable to keep on top of it. This causes many important emails to be buried amongst less important ones. For some this is a significant source of stress (Barley, Meyerson, \& Grodal, 2011). Furthermore, time spent managing email can be distracting and impact negatively on other important activities (Boswell \& Olsen-Buchanan, 2007).

In their seminal paper, Whittaker and Sidner (1996) identified several strategies that people use to manage their inbox. These included "no filers", people who never managed their inbox, "frequent filers", people who manage their inbox daily, and "spring cleaners", people who manage their inbox intermittently (every 1-3 months). Surprisingly, since this seminal paper was published it is still not clear which of these strategies is best.

We examined whether using different email management strategies changes the total time that is spent on email. By recording the amount of time people spend on email using different strategies, we attempt to identify which strategy is the most efficient. We assume that the most efficient strategy is the one that requires users to spend the least amount of time in order to manage their email inbox. The two email management strategies that we investigate are modifications of the "frequent filing" and "spring cleaning" strategies.

University academics were recruited as participants because they tend to receive a high volume of email everyday. They were required to spend a week as "frequent filers" and a week as "spring cleaners". As "frequent filers", participants processed email (by responding, filing or deleting it) throughout the day. This strategy will be referred to as the "frequent" email management strategy. As "spring cleaners", participants attempted to leave as much of their email processing as possible to single session each day. This strategy will be referred to as the "once a day" email management strategy. The amount of time spent on email in each condition was recorded using a commercially available software tool called Rescue Time (www.rescuetime.com). This software runs in the background on the participant's computer, tracking the time they spend actively using a specific 
application or website. The participant's subjective feelings about each strategy were also recorded.

In the following section we review work on email overload, email related stress and the cost of task switching due to email. As will become clear, a once a day email management strategy should be better than a frequent checking strategy.

\section{RELATED WORK}

\subsection{How do people manage email?}

Whittaker and Sidner (1996) investigated how people use email in an office environment. They identified two main issues that users had with email: (1) they struggled to read and respond to emails in a timely manner; and (2) they had difficulty retrieving information from old emails that were needed at a later time. Both of these issues were due to users receiving emails at a greater rate than they could deal with them. These problems were found to cause reduced responsiveness to emails and important information being lost amongst users' inboxes.

Whittaker and Sidner (1996) identified three distinct email management strategies. These have been referred to as no filers, spring cleaners and frequent filers. No filers tend not to file their emails but instead let them accumulate in the inbox. Spring cleaners manage their inbox intermittently every few months. They experience 'tidy' inboxes for short periods after a clean up but generally have overloaded inboxes. In contrast, frequent filers dedicate time to keeping their inbox as empty as possible by filing and deleting emails on at least a daily basis. This results in a small and wellorganised inbox containing predominantly unread messages.

While previous research has attempted to reveal the strategies users adopt in order to manage their email inbox, no research has investigated which, if any, of these strategies is best. Although the amount of time spent on email is a key factor in assessing what makes an email strategy better or worse, it is also important that an email strategy decreases the amount of stress a user experiences due to their email inbox.

\subsection{The cost of frequent email checking}

One way to assess the effectiveness of an email strategy is in terms of how distracting it is. If a strategy requires users to frequently switch from their current activity to manage their email inbox then it is likely that the user will be less productive in completing their main task. For instance, Venolia Dabbish, Cadiz, and Gupta (2001) observed that many people keep their email visible at least twothirds of the time during their work day, so as to be able to regularly check on the status of their inbox. Similarly, Jackson, Dawson and Wilson (2001) found that many users check for new email every five minutes. These checks were to assess whether any new emails in their inbox required urgent attention. However, very few emails are of such high importance that they require immediate attention. What then is the cost to the other ongoing activities that the person is engaged in?

It is well known that interruptions are disruptive and take time to recover from. Although some interruptions can be beneficial, for example they can allow a user to gain relevant information to their current task (Mark, González and Harris, 2005), in most cases interruptions are distracting and reduce productivity (Perlow, 1999). Because emails do not require immediate action and can be ignored until a user has the free time available to handle them, it is possible that they will be less distracting than other forms of communication. However, Jackson et al. (2001) found that $70 \%$ of emails were reacted to within 6 seconds and $85 \%$ within 2 minutes. Furthermore, each time email was checked there was an average of 64 seconds taken to return to the previous task. It has been suggested that this estimate is actually conservative and that users actually take many minutes to return to their original task after managing and responding to their emails (González \& Mark, 2004). This suggests that frequently checking email can be very detrimental to other on-going activities.

\subsection{Email as a stressor}

We are also interested in how people feel about using each email management strategy. It is known that people levels of stress increase with the time spent on email (e.g., Barley et al., 2011). This is because spending a lot of time on email can cause an individual's workday to be longer. Coupled with this is the fact that people can continue to work on email at home during periods that traditionally would have been set aside for other activities (Boswell \& Olsen-Buchanan, 2007). Therefore if one of the strategies used in the current study results in participants spending less time on email, then we might expect this strategy to reduce feelings of overload and work-related stress.

\subsection{Overview of the current study}

The focus of the current study is to determine whether a once a day email management strategy is better than a frequent checking strategy. The previous work discussed above suggests that there should be several benefits to adopting a once a day strategy. First, people should be more efficient in processing their inbox. In particular, they should be more likely to process all of the unread emails in their inbox, rather than deferring the processing of 
some emails until later. Second, adopting a once a day strategy should reduce the frequency with which people interrupt themselves to check on their email inbox. Finally, due to a decrease in the frequency which individuals check their email, they should feel less stressed due to email being less intrusive into their lives throughout the day thus enabling more time to be spent on other activities uninterrupted.

There are also several potential issues with adopting a once a day email strategy. First, individuals might worry that this strategy will result in them falling behind on their email. Second, participants might find it stressful trying to process all of their emails in a single session. Third, despite email being an asynchronous method of communication, many emails exchange information that is highly time sensitive. Even a fast reply merely acknowledging receiving an email is sometimes preferred to a slow reply. These problems raise questions of whether participants will be capable of only checking their emails once a day, how they will feel about this restriction being placed on them, and what implications these restrictions will have on their experience at work. However, it should be noted that recent work by Mark, Voida, \& Cardello (2012) has shown that people can survive without email and still complete work activities - because they find other ways to communicate.

To evaluate whether there are benefits to a particular email management strategy we had a group of busy university academics, all of who received a lot of email every day, try and adopt a once a day email management strategy for a week. We had the same individuals also try and adopt a frequent email management strategy for a week. We were interested in whether this change in email management strategy would have an effect on the amount of time spent on email. We were also interested in gathering subjective reports of how people felt about each email management strategy.

\section{METHOD}

\subsection{Participants}

An opportunistic sample of seven (three female) junior academics $(1 \times$ lecturer, $1 \times$ research fellow, $5 \times$ graduate research students) took part in the study, with a mean age of 28 years $(S D=4.1$, range $=24-34)$.

All of the participants reported that they received a large number of emails and that they felt that they struggled to manage their inbox effectively. In exchange for their participation, participants were given feedback on which email management strategy was more efficient for them as well as information on how much time they were spending on email during the course of the study.

\subsection{Design}

We gave instructions for participants to try and adopt either a once a day or a frequent email management strategy. For the once a day email management strategy, participants were asked to limit how often they checked their email inbox and process most of their email in a single session each day. We did also tell participants that they could reply to urgent or important emails whenever they needed to. For the frequent email management strategy, participants were encouraged to frequently check their email inbox throughout the day and to process all emails as soon as possible with the explicit goal of maintaining a relatively empty/low inbox.

We used a within-subjects design so that every participant had to try and adopt each email management strategy for a week. The order in which each strategy was used was counterbalanced across participants.

We were interested in whether participants changed how frequently they checked email depending on which strategy they were supposed to be following. Assuming that there is a difference in email behaviour, we wanted to find out which strategy led to participants spending the least amount of time on email over the week. We were also interested in getting a general sense of how participants felt about each email management strategy.

\subsection{Materials}

We used a software tool called RescueTime (www.rescuetime.com) to record how much time participants spent on email. RescueTime records the time that people spend actively using an application on their computer, giving an hourly breakdown of usage for each application. By using RescueTime's "white-list" feature, participants gave the experimenter remote access to their usage data for email applications and websites.

To get a sense of how participants felt about following each email management strategy, a brief questionnaire was developed and put on Survey Monkey (www.surveymonkey.com). The questions asked were: (1) How similar to your current email strategy is the strategy used? (2) How easy was it to adopt the strategy? (3) How strictly did you follow the instructions of the strategy? (4) How long do you think you spent on email compared to a normal week? (5) How stressed did you feel by email whilst using this strategy? (6) How distracted did you feel by email whilst using this strategy? (7) How well have you been able to manage your inbox whilst using this strategy? Responses were 
given on a 9-point Likert scale, where higher valued responses represent a stronger level of agreement to the question.

\subsection{Procedure}

Participants were briefed about the experiment before consenting to participate. An automated email was sent to participants from the experimenter that contained instructions explaining how to install RescueTime on their computers. Participants were asked to provide information on which websites or programs they used for email services so that the usage of these could be tracked. They were also asked to provide a brief summary of their current email management strategies.

Participants spent a week using each of the two email management strategies. While doing this, participants were encouraged to keep a diary of any difficulties, feelings or comments they had about following the email management strategy. At the end of each week, participants were prompted to complete a brief online questionnaire about their experience of following the email management strategy. The following week, participants used the alternate strategy and completed the same diaries and questionnaires as they did for the first week.

\section{RESULTS}

If participants followed the instructions to change their email management strategy from one week to the next, then we would expect to see a change in the number and duration of email sessions (that is, each unique time that an email application or website was used).

Participants clearly struggled to implement the once a day strategy: the mean number of email sessions per week was 25 (i.e., about 3.6 sessions per day). However, there is evidence that the participants were changing their email behaviour across conditions because the mean number of sessions in the frequent checking condition was 56 (i.e., 8 sessions per day).

Given that participants were checking email less frequently when following the once a day strategy, we next consider whether this brought about a reduction in the amount of time spent on email during the week. It was found that participants spent fewer minutes on email over the course of the week when following the once a day email management strategy $(M=148.29 \mathrm{~min}, S D=$ $108.88 \mathrm{~min}$ ) compared to the frequent email management strategy $(M=256.71 \mathrm{~min}, S D=$ $237.65 \mathrm{~min})$. Because of the small sample size $(N=7)$ and high degree of variance in the amount of time spent on email between participants, we use a non-parametric Wilcoxon signed-rank test to
Table 1. Responses to questionnaire items after using each email management strategy for a week (mean and standard deviation). Responses are given on a 9-point Likert scale, where higher valued responses represent a stronger level of agreement to the question.

\begin{tabular}{llll}
\hline Questionnaire item & \multicolumn{2}{c}{ Strategy used } & Z-Ratio \\
\cline { 2 - 3 } & $\begin{array}{c}\text { Once a } \\
\text { day }\end{array}$ & Frequent & \\
\hline $\begin{array}{l}\text { How similar to your } \\
\text { current email strategy is } \\
\text { the strategy used? }\end{array}$ & $7.7(1.8)$ & $2.7(2.2)$ & $2.21^{*}$ \\
\hline $\begin{array}{l}\text { How easy was it to } \\
\text { adopt the strategy? }\end{array}$ & $8.1(0.4)$ & $2.6(2.4)$ & $2.38^{*}$ \\
\hline $\begin{array}{l}\text { How strictly did you } \\
\text { follow the instructions of } \\
\text { the strategy? }\end{array}$ & $4.9(2.0)$ & $2.1(1.3)$ & $2.12^{*}$ \\
\hline $\begin{array}{l}\text { How long do you think } \\
\text { you spent on email } \\
\text { compared to a normal } \\
\text { week? }\end{array}$ & $5.0(1.5)$ & $4.9(0.7)$ & 0.11 \\
\hline $\begin{array}{l}\text { How stressed did you } \\
\text { feel by email whilst } \\
\text { using this strategy? }\end{array}$ & $5.4(1.3)$ & $4.7(1.3)$ & 0.00 \\
\hline $\begin{array}{l}\text { How distracted did you } \\
\text { feel by email whilst } \\
\text { using this strategy? }\end{array}$ & $5.7(1.6)$ & $5.6(2.0)$ & 0.92 \\
\hline $\begin{array}{l}\text { How well have you been } \\
\text { able to manage your } \\
\text { inbox whilst using this } \\
\text { strategy? }\end{array}$ & $5.1(2.0)$ & $5.1(1.2)$ & 0.17 \\
\hline $\begin{array}{l}\text { Note *p<.05 } \\
\text { tom }\end{array}$ & & & \\
\hline
\end{tabular}

test for statistical significance. Results show that participants spent significantly less time on email when following the once a day email management strategy compared to the frequent email management, $Z=2.20, p=.03$.

Finally, we consider the questionnaire data. Table 1 shows the mean responses given by participants to each question after using each email management strategy. A series of Wilcoxon signed-rank tests were performed to see if there were significant differences in response patterns between the two conditions. It can be seen that participants rated the frequent email management strategy as significantly more similar to their usual email strategy, significantly easier to adopt and significantly more strictly followed compared to the once a day email strategy. However, following the once a day strategy did not lead to significant reduction in how participants felt about the amount of time they were spending on email, how distracted and stressed they were by email, or how well they felt they were able to mange their inbox.

\section{DISCUSSION}

The current study investigated the effect of using two different email strategies on the amount of time spent on email as well as on subjective self- 
reported measures, such as stress and level of distraction caused by email. Firstly, it was found that the manipulation of email strategy did have an effect on the participant's behaviour regarding email. When using a once a day strategy, participants checked mail less frequently and spent less time on email when they were following a frequent email management strategy. Participants used fewer but longer sessions in order to handle their email in the once a day management strategy as opposed to more regular and shorter sessions in the frequent email management strategy. This suggests that participants would spend less time task switching, therefore avoiding the cost associated with it identified by previous research (Perlow, 1999).

It was hypothesised that by using a strategy that required participants to manage their email inbox only once a day participants would spend less time on email than if they used a strategy in which they frequently managed their email inbox. The results of this study support this idea and show that participants spent less time on email when following the once a day email management strategy compared to the frequent checking strategy. These results therefore suggest that a once a day email management strategy might lead people to spend less time each day on email.

Another key aspect in assessing the quality of an email strategy is the ease with which it is adopted. Participants reported finding it more difficult to adopt the once a day email management strategy than the frequent email management strategy. How easily an email management strategy was adopted was correlated with how strictly participants reported following the instructions of the strategy. This suggests that if a strategy is too difficult to adopt it is not likely to be followed very strictly. The ease of adoption of the strategy and how strictly it was followed were both correlated with how similar a strategy was to a participant's usual email strategy. Therefore, it is suggested that the reason the once a day email management strategy was difficult to adopt (and therefore less strictly followed) is not because of something inherent to the strategy itself but due to it being more different to the participant's usual management strategy. This was supported by the qualitative data provided by participants explaining that their usual strategy was to manage emails as they arrive.

It was also hypothesised that using a once a day email management strategy could reduce the amount of stress participants experienced due to email. The basis of this hypothesis was that spending less time on email reduces stress (Barley et al., 2011). The strategy used by participants did not result in a significant difference in reported stress levels or the amount of time that participants spent on email. Although at first this seems like a negative result there is a positive to be drawn from it in that participants did not feel more stressed by adopting the once a day email management strategy.

\subsection{Implications}

The finding that participants could spend less time on email by using a once a day email management strategy has productivity implications. If an effective way to implement a once a day email management strategy could be found that avoids the drawbacks identified in Mark et al. (2012), such as feelings of isolation and fear of being overwhelmed by emails, then employees could spend less time managing their email inboxes and more time working on other tasks.

The finding that an email strategy less similar to a user's normal email strategy is harder to adopt and less likely to be followed strictly suggests that in order to encourage users to adopt a new email management strategy it would be beneficial to start with small deviations away from their normal strategy and gradually move towards larger changes.

\subsection{Limitations}

The most critical limitation of the study is the small sample size. Recruiting participants was difficult due to the reluctance of users to change their email management strategy to a once a day strategy. This reluctance existed as a result of user's fears that they would not be able to stay on top of their email inbox and would become overwhelmed if they allowed emails to accumulate in their inbox. This kind of concern is common and has been expressed by participants of several other studies (e.g. Barley et al., 2011).

RescueTime could not be installed on iPhones or iPads and therefore email usage times on them could not be recorded. This caused a problem because some participants usually used their iPhones or iPads as part of their email management strategy. Ideally only participants who did not use these devices would be selected for participation, however due to the previously mentioned problems with recruiting enough participants, this was not possible. The best effort to minimise the effect of this limitation was to request that participants attempt to avoid using their iPhone or iPad for email. One way this was encouraged was suggesting users to turn off push email in order to reduce the kneejerk reaction of checking emails on a mobile device as soon as they arrive. Overall participants reported success in avoiding using their phones for email.

A further limitation was that all time spent on an email application was logged under the same title. This meant that it was not possible to discriminate 
between the times when participants were using an email application in order to manage their email inbox and when they were using it for other things. For example one participant reported the following:

"Today I used my mail application about three times when I wasn't specifically checking emails. Rather I was carrying out a task on my to-do list that involved the use of my email application: for example, a colleague sent me some files for a lecture I was preparing via his public Dropbox folder, which I opened via mail, and I spent a while organising the session chairs for a conference I'm chairing also using email."

Although on the whole participants were fairly strict in following each strategy as they were instructed, multiple participants reported deviating slightly from the instruction they received, particularly in the once a day management strategy. This could be prevented in future studies by offering participants financial incentives for following the strategies very strictly.

Future studies could improve on the current study by testing the measures from the questionnaire in improved ways. For example, to get an improved measure of the effect of each email strategy on stress levels, skin conductance could be used, or measures of coping and overload as done by Barley et al. (2011). To measure the effect of email strategy on how distracted a participant is by email software that records window changes could be used or an observer could be present to record the time taken to resume tasks, similar to the method used by Mark et al. (2012).

\section{CONCLUSION}

The results of this study suggest that adopting a once a day email processing strategy is beneficial in terms of reducing the amount of time that is spent on email. This is because email can be more efficiently worked on in single, longer sessions rather than by nibbling at it throughout the day. Surprisingly though this reduction in time spent on email from following the once a day strategy did not lead to a reduction in email-related stress, or even the perception of how much time is being spent on email. This is likely because most of our participants were already using an email management strategy quite similar to a once a day strategy to manage their email.

\section{AKNOWLEDGEMENTS}

This work was undertaken as the first author's BSc research project at UCL and is part of the Digital Epiphanies project, supported by the UK Engineering and Physical Sciences Research Council [EP/K025392/1].

\section{REFERENCES}

Barley, S.R., Meyerson, D.E., \& Grodal, S. (2011). E-mail as a source and symbol of stress. Organization Science, 22, 887-906.

Boswell, W.R., \& Olson-Buchanan, J.B. (2007). The use of communication technologies after hours: The role of work attitudes and work-life conflict. Journal of Management, 33, 592-610.

Crystal, D. (2001). Language and the Internet. Cambridge University Press, Cambridge UK.

González, V.M., \& Mark, G. (2004). Constant, constant, multi-tasking craziness: managing multiple working spheres. In Proceedings of the SIGCHI Conference on Human Factors in Computing Systems (CHI '04), 113-120. ACM, New York, NY, USA.

Jackson, T., Dawson, R., \& Wilson, D. (2001). The cost of email interruption. Journal of Systems and Information Technology, 5, 81-92.

Mark, G., González, V., and Harris, J. (2005). No task left behind? Examining the nature of fragmented work. In Proceedings of the SIGCHI Conference on Human Factors in Computing Systems (CHI '05), 321-330. ACM, New York, NY, USA.

Mark, G., Voida, S., \& Cardello, A. (2012). A pace not dictated by electrons: an empirical study of work without email. In Proceedings of the SIGCHI Conference on Human Factors in Computing Systems (CHI '12), 555-564. ACM, New York, NY, USA.

Perlow, L.A. (1999). The time famine: towards a sociology of work time. Administrative Science Quarterly, 44, 57-81.

Venolia, G.D., Dabbish, L., Cadiz, J.J., \& Gupta, A. (2001). Supporting email workflow. Microsoft Research.

Whittaker, S. \& Sidner, C. (1996). Email overload: exploring personal information management of email. In Proceedings of the SIGCHI Conference on Human Factors in Computing Systems $(\mathrm{CHI}$ '96), 276-283. ACM, New York, NY, USA.

Whittaker, S., Bellotti, V., \& Gwizdka, J. (2006). Email in personal information management. Communications of the ACM, 49, 68-73. 